\title{
Insight into Highly Conserved H1 Subtype-Specific Epitopes in Influenza Virus Hemagglutinin
}

\author{
Ki Joon Cho ${ }^{1 *}$, Kwang W. Hong ${ }^{2}$, Se-Ho Kim ${ }^{2 \star}$, Jong Hyeon Seok ${ }^{1}$, Sella Kim ${ }^{1}$, Ji-Hye Lee ${ }^{1}$, \\ Xavier Saelens ${ }^{3,4}$, Kyung Hyun Kim ${ }^{1 *}$
}

1 Department of Biotechnology \& Bioinformatics, Korea University, Sejong, Korea, 2 Antibody Engineering Laboratory, Central Research Center, Green Cross Corp., Yongin Kyunggi, Korea, 3 VIB Inflammation Research Center, Ghent, Belgium, 4 Department of Biomedical Molecular Biology, Ghent University, Ghent, Belgium

\begin{abstract}
Influenza viruses continuously undergo antigenic changes with gradual accumulation of mutations in hemagglutinin (HA) that is a major determinant in subtype specificity. The identification of conserved epitopes within specific HA subtypes gives an important clue for developing new vaccines and diagnostics. We produced and characterized nine monoclonal antibodies that showed significant neutralizing activities against $\mathrm{H} 1$ subtype influenza viruses, and determined the complex structure of HA derived from a 2009 pandemic virus A/Korea/01/2009 (KR01) and the Fab fragment from H1-specific monoclonal antibody GC0587. The overall structure of the complex was essentially identical to the previously determined KR01 HA-Fab0757 complex structure. Both Fab0587 and Fab0757 recognize readily accessible head regions of HA, revealing broadly shared and conserved antigenic determinants among $\mathrm{H} 1$ subtypes. The $\beta$-strands constituted by Ser $110-\mathrm{Glu} 115$ and Lys169-Lys170 form H1 epitopes with distinct conformations from those of H1 and H3 HA sites. In particular, Glu112, Glu115, Lys169, and Lys171 that are highly conserved among $\mathrm{H} 1$ subtype HAs have close contacts with HCDR3 and LCDR3. The differences between Fab0587 and Fab0757 complexes reside mainly in HCDR3 and LCDR3, providing distinct antigenic determinants specific for 1918 pdm influenza strain. Our results demonstrate a potential key neutralizing epitope important for $\mathrm{H} 1$ subtype specificity in influenza virus.
\end{abstract}

Citation: Cho KJ, Hong KW, Kim S-H, Seok JH, Kim S, et al. (2014) Insight into Highly Conserved H1 Subtype-Specific Epitopes in Influenza Virus Hemagglutinin. PLoS ONE 9(2): e89803. doi:10.1371/journal.pone.0089803

Editor: Eliane Namie Miyaji, Instituto Butantan, Brazil

Received August 10, 2013; Accepted January 27, 2014; Published February 26, 2014

Copyright: (C) 2014 Cho et al. This is an open-access article distributed under the terms of the Creative Commons Attribution License, which permits unrestricted use, distribution, and reproduction in any medium, provided the original author and source are credited.

Funding: The authors wish to acknowledge the technical support from the staffs at beamlines of Pohang Light Source (5C) and PF (BL-17A) synchrotrons. This work was supported by grants from the Mid-career Researcher (KHK 2010-0029242) and Basic Research (KJC 2012-044524) Programs through NRF funded by the ME, the Transgovernmental Enterprise for Pandemic Influenza in Korea (KHK 2011-A103001), Korea University (KJC), and the BK21 plus program of the ME, Korea. The funders had no role in study design, data collection and analysis, decision to publish, or preparation of the manuscript.

Competing Interests: KWH is currently, and SHK was at the time of the study, an employee of Green Cross Corp., Korea. There are no patents, products in development or marketed products to declare. This does not alter the authors' adherence to all the PLOS ONE policies on sharing data and materials.

*E-mail: c-azna@korea.ac.kr (KJC); khkim@korea.ac.kr (KHK)

a Current address: University-Industry Cooperation Foundation, Kangwon National University, Chuncheon, Kangwon-Do, Korea

\section{Introduction}

Influenza is a viral infectious disease of the respiratory tract that affects millions of people annually [1]. Combined with subsequent infection from bacterial pneumonia, influenza remains one of the leading causes of death in many countries [2]. The 1918 influenza pandemic (pdm) killed 40-50 million people worldwide [3] and the 2009 pdm influenza that was identified in 214 countries caused more than 18,000 deaths worldwide, despite global influenza preparedness [4].

Influenza viruses which belong to the family of Orthomyxoviridae have three antigenically distinct types of virus, A, B, and C $[5,6]$. Influenza A viruses contain three surface proteins: hemagglutinin (HA), neuraminidase (NA), and a proton channel M2 [7,8]. The viruses are divided into subtypes on the basis of differences in the antigenicity of HA and NA. After the recent discovery of a new subtype virus genome identified from bat, there are currently 17 HA subtypes (H1-H17) and 10 NA subtypes (N1-N10) known $[9,10]$. Influenza A viruses with three $\mathrm{HA}(\mathrm{H} 1, \mathrm{H} 2$ and $\mathrm{H} 3)$ and two NA (N1 and N2) serotypes have adapted to humans to produce H1N1 pdm in 1918 and 2009, H2N2 pdm in 1957, and H3N2 pdm in 1968 [11-13].
The 2009 pdm viruses were derived from a reassortment of six gene segments from triple reassortant swine virus and two gene segments from Eurasian influenza A $(\mathrm{H} 1 \mathrm{Nl})$ swine virus lineage [13]. Amino acid sequence identity between 2009 pdm HA and those derived from previous vaccine strains such as A/Brisbane/ 59/07 (H1Nl) and A/Solomon Islands/3/2006 (H1Nl) reaches approximately $80 \%$, which drops to $35-40 \%$ within the antigenic sites. It was shown that the antigenic and glycosylation patterns of 2009 pdm HA are rather similar to those of 1918 pdm HA, showing 20\% amino acid difference in the antigenic sites [14].

$\mathrm{HA}$ is synthesized as a precursor, HA0, that trimerizes in the endoplasmic reticulum and is transferred through the Golgi apparatus to the cell surface [15-17]. Cleavage of the precursor HA into the subunits HAl and HA2 by a cellular protease is required for viral infectivity [18,19]. The HA2 stem region, proximal to the viral membrane, is highly conserved across strains and among most subtypes. Since the first cross-neutralizing antibody against influenza virus was reported [20], many structures of broadly neutralizing antibodies in complex with HA proteins have been determined [21-24]. Several studies also reported the existence of HA subtype-specific and inter subtype- 
conserved epitopes [25-27]. However, immune specific epitopes in H1N1 influenza virus have not been completely assessed.

During the production of $\mathrm{Hl}$-specific monoclonal antibodies against 2009 pdm H1Nl strains, we isolated and characterized nine $\mathrm{H} 1$-specific monoclonal antibodies which neutralized a broad range of $\mathrm{Hl}$ subtype influenza viruses. Among them, we determined the structure of the HA protein from a 2009 pandemic virus A/Korea/01/2009 (KR01) in complex with the Fab fragment from GC0587 and compared with the KR01 HAFab757 complex structure. In addition to GC0587, GC0757 exhibits additional activity against A/Brevig Mission/1/1918. The structural features of the complexes provide a understanding of how antibodies with subtype specificity can distinguish antigenic determinants.

\section{Results}

\section{In vivo Production and in vitro Characterization of Monoclonal Antibodies}

In our initial immunogenicity experiment, A/California/07/ 2009 (CA07) was used to immunize groups of BALB/c mice and antibody-producing cells were screened by ELISA for secretion of antibodies. Nine monoclonal antibodies were then tested for neutralization against a panel of 14 isolates including $\mathrm{H} 1$, 2009 pdm H1, H2, H3, H5, and H7 (Table 1). Among them, four monoclonal antibodies GC0587, GC0757, GC1517, and GC1761 were shown to neutralize a broad range of $\mathrm{H} 1$ subtype influenza A viruses including 2009 pdm isolates. GC0587, GC0757 and GC1517 exhibited significant neutralizing activity against 2009 pdm H1Nl strains and a seasonal H1Nl strain, whereas GC0757, GC1517 and GC1761 showed the activity against A/Brevig Mission/1/1918.

Examination based on ELISA assays for the antigenicity against KR01 HA revealed that GC0346, GC0587, GC0757, and GC1517 had significantly high affinity to HA (Fig. 1), whereas GC0352, GC1245, GC1289, GC1747, and GC1761 showed moderate or little affinity even at high concentrations. In particular, GC0587 and GC0757 showed high affinity binding to HAs derived from both KR01 and CU44 strains and their Fab fragments were found to bind to HAs derived from $\mathrm{H} l$ subtype viruses with high affinities $\left(\mathrm{K}_{\mathrm{d}} 2.5-8.9 \mathrm{nM}\right)$. The dissociation constants were within the range typically required for effective neutralization, comparable to stem binding antibodies $\left(\mathrm{K}_{\mathrm{d}}\right.$ $\sim 10 \mathrm{nM})$ [21]. In order to study broad antigenicity among $\mathrm{H} 1$ subtype strains, we selected four antibodies GC0346, GC0587, GC0757, and GC1517 and attempted to crystallize their Fab fragments in complex with KR01 HA. Our attempt yielded crystals derived from antibodies GC0587 or GC0757 only, suitable for X-ray structural determination.

\section{Overall Structure of the KR01 HA-Fab0587 Complex}

The purified recombinant KR01 HA was monomeric in solution. The structure from small rhombic crystals $(0.02 \times 0.02 \times 0.05 \mathrm{~mm})$ of KR01 HA-Fab0587 complex was determined at $3.1 \AA$ resolution (Table 2). The asymmetric unit of the crystal contained four copies of HA-Fab0587 complexes, where Fab binds to the head region of KR01 HA resulting in a linear arrangement of the complex consisting of one Fab, two head regions of HA, and another Fab molecule (Fig. 2A). The HAFab0587 complex structure revealed head-to-head arrangement of HA as shown in KR01 HA (PDB ID: 4EDA) and the HA-Fab0757 complex (r.m.s.d. was $1.3 \AA$ ), to which the longitudinal axis of Fab in the complex was parallel. The overall arrangement of HA and Fab0587 was thus very similar to that of the HA-Fab0757 complex structure [28]. Superposition of the two complex structures yielded an overall r.m.s.d. of $2.1 \AA$, whereas the head or stem regions superimposed well individually $(0.9$ and $0.8 \AA$ on average, respectively). The stem region was largely invisible in the electron density map, for which a partial model could be built, consisting of residues 414-427 (87-100 of HA2) in loop and $\alpha$-helical conformations with high $\mathrm{B}$ factors ranging from 54-128 $\mathrm{A}^{2}$. A possible orientation of the HA2 domain can be derived from this partial model. When the KR01 HA structure was superimposed to that of the HA-Fab0587 complex, the head region of KR01 HA

Table 1. In vitro neutralization activity of antibodies against a panel of HA from influenza A viruses.

\begin{tabular}{|c|c|c|c|c|c|c|c|c|c|}
\hline Antibody HA from Strains* & GC 0346 & GC 0352 & GC 0587 & GC 0757 & GC 1245 & GC 1289 & GC 1517 & GC 1747 & GC 1761 \\
\hline A/Brisbane/59/2007(H1N1) & - & - & ++ & ++ & - & - & ++ & - & + \\
\hline A/California/04/2009(H1N1) & ++ & + & ++ & ++ & - & - & ++ & - & ++ \\
\hline A/Brevig Mission/1/1918(H1N1) & - & - & - & ++ & - & - & ++ & - & ++ \\
\hline A/California/07/2009(H1N1) & ++ & ++ & ++ & ++ & - & - & ++ & - & ++ \\
\hline A/Japan/305/1957(H2N2) & - & - & - & - & - & - & ++ & - & - \\
\hline A/Brisbane/10/2007(H3N2) & - & - & - & - & - & - & - & - & - \\
\hline A/Anhui/1/2005(H5N1) & - & - & - & - & - & - & - & - & - \\
\hline A/Indonesia/5/2005(H5N1) & - & - & - & - & - & - & - & - & - \\
\hline A/Viet Nam/1194/2004(H5N1) & - & - & - & - & - & - & - & - & - \\
\hline A/Viet Nam/1203/2004(H5N1) & - & - & - & - & - & - & - & - & - \\
\hline A/bar-headed goose/Qinghai/14/2008 (H5N1) & - & - & - & - & - & - & ++ & - & - \\
\hline A/turkey/Turkey/1/2005(H5N1) & - & - & - & - & - & - & - & - & - \\
\hline A/Netherlands/219/03(H7N7) & - & - & - & - & - & - & - & - & - \\
\hline 2009 pandemic vaccine & ++ & ++ & ++ & ++ & ++ & + & ++ & + & ++ \\
\hline
\end{tabular}

+represents a positive neutralization activity of each antibody determined by ELISA and -represents a negative neutralizing activity.

${ }^{*} \mathrm{~A} /$ California/04/2009(H1N1), A/Brisbane/59/2007(H1N1), A/California/04/2009(H1N1), A/Brevig Mission/1/1918(H1N1), A/California/07/2009(H1N1), A/Japan/305/

1957(H2N2), A/Brisbane/10/2007(H3N2), A/Anhui/1/2005(H5N1), A/bar-headed goose/Qinghai/14/2008(H5N1), A/lndonesia/5/2005(H5N1), A/turkey/Turkey/1/ 2005(H5N1), A/Viet Nam/1194/2004(H5N1), A/Viet Nam/1203/2004(H5N1), A/Netherlands/219/03(H7N7).

doi:10.1371/journal.pone.0089803.t001 

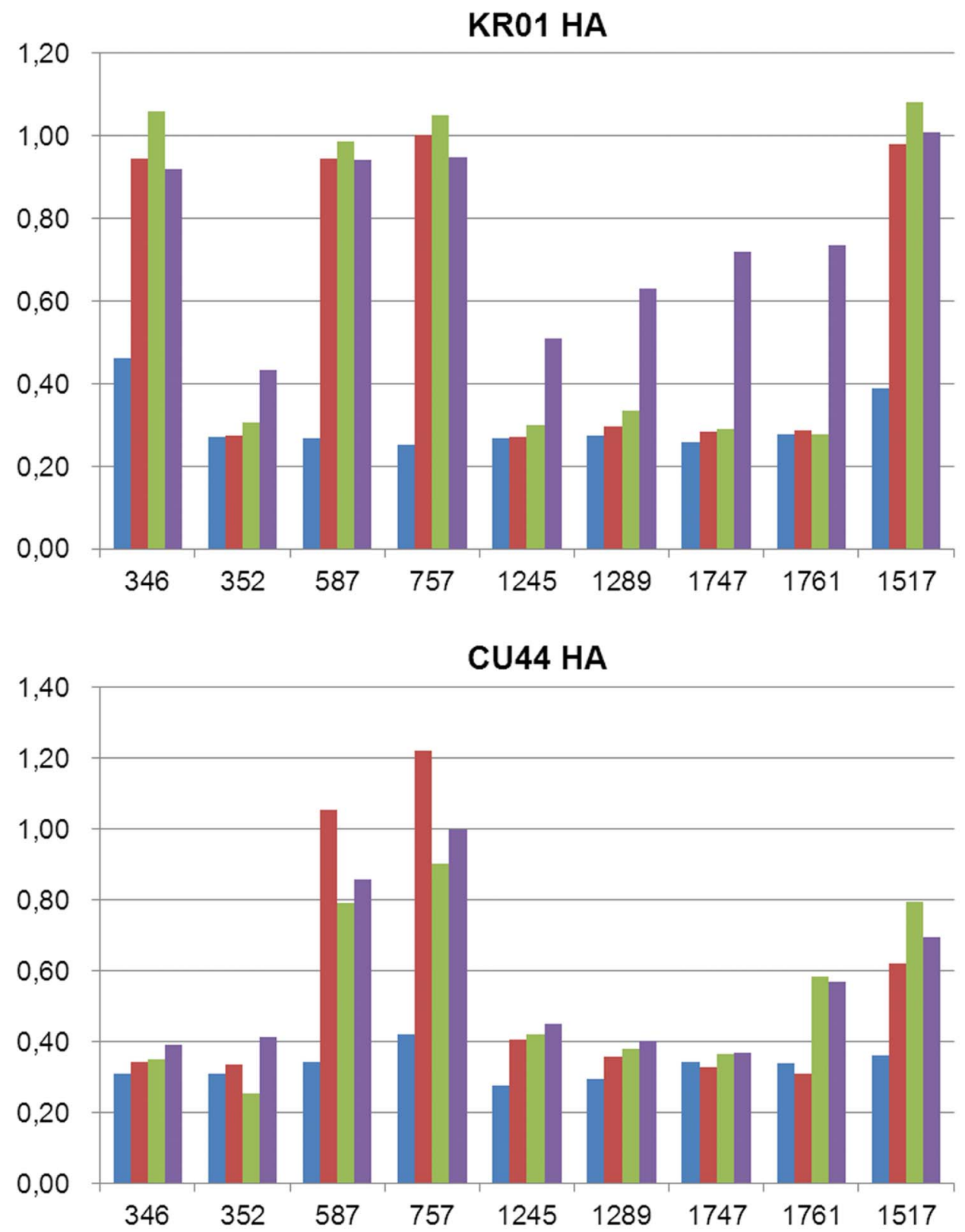

\section{$0 \mu \mathrm{g} / \mathrm{ml} \square 0.1 \mu \mathrm{g} / \mathrm{ml} \square 1.0 \mu \mathrm{g} / \mathrm{ml} \square 10.0 \mu \mathrm{g} / \mathrm{ml}$}

Figure 1. Binding affinities of monoclonal antibodies against H1 HAs. ELISA analysis results of monoclonal antibodies against KR01 and CU44 HAs that were coated on the 96-well plates to which 0 to $10 \mu \mathrm{g} / \mathrm{ml}$ of antibodies were added. Absorbance was measured after addition of TMB solution to each well at $490 \mathrm{~nm}$.

doi:10.1371/journal.pone.0089803.g001

was found to be rotated clockwise by $\sim 20^{\circ}$ relative to the stem region (Fig. 2B). We observed that there are empty spaces in the crystal lattice for which the HA2 domain can exist (Figure S1 in File S1). The conformation of the stem region is known to be metastable, which is temporarily stabilized by the head region of capped conformation [29,30]. The conformational changes of HA that alleviate the constraints imposed in the pre-fusion state are known to provide the energy that is required to induce membrane fusion. The KR01 HA-Fab0587 complex structure demonstrates that the head region of HA molecule is stabilized by antibody binding and the invisible stem region adopts different orientations relative to the head region from what we have observed in typical HA proteins (Fig. 2C).

\section{A conserved H1 Subtype-specific Epitope of HA}

The amino acid sequence identity between the variable domains of GC0587 and GC0757 is 93\% in the H-chain and 98\% in the Lchain (Fig. 3A), with the largest difference in HCDR3. The interface in the HA-Fab complexes was found to consist of interactions with heavy chain CDRs (HCDRs) 2 and 3 and light 


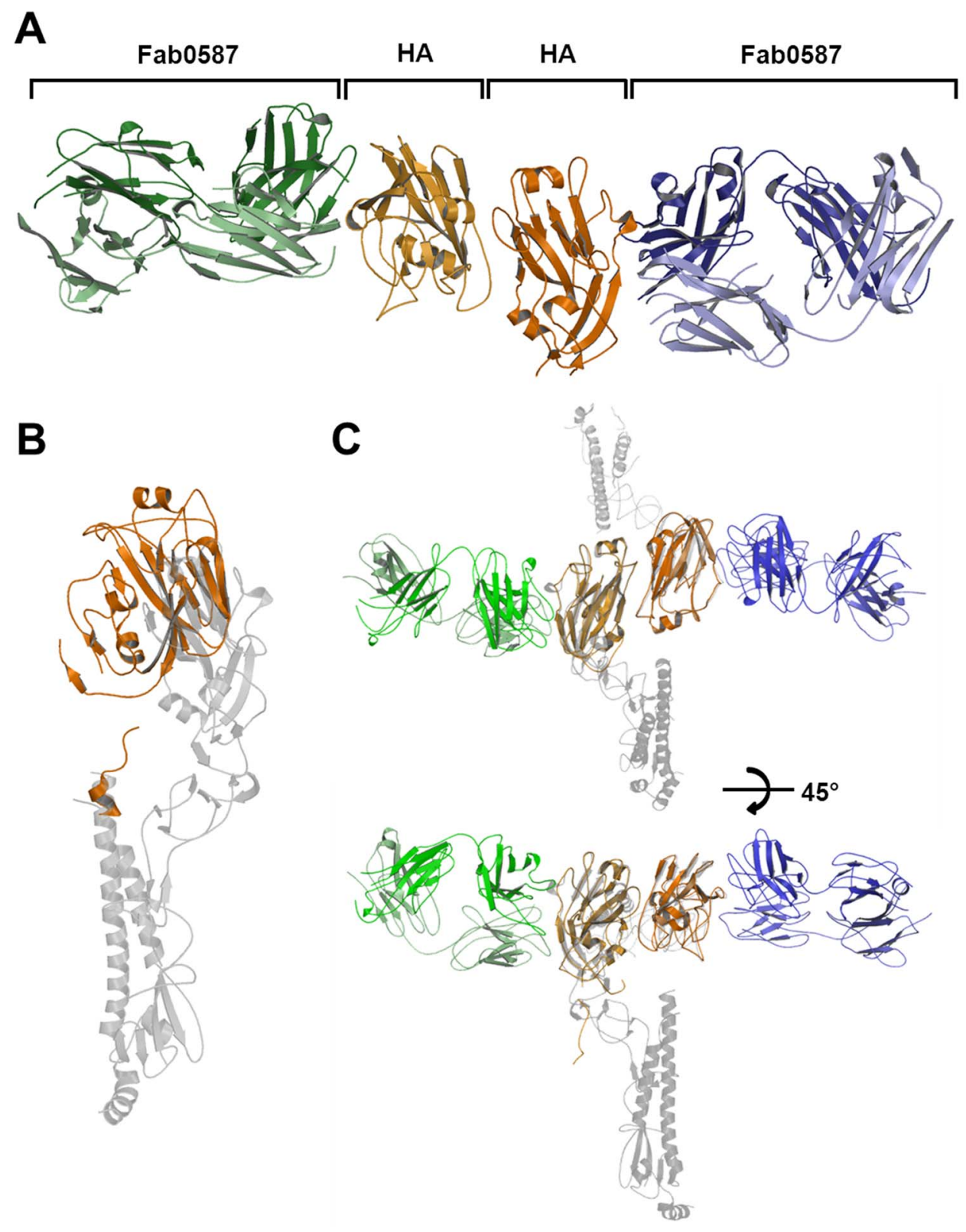

Figure 2. Overview of the structures of KR01 HA and its complex with Fab0587. (A) Structure of KR01 HA-Fab0587 complex. HA are colored in orange and light brown, H-chains are blue and green, and L-chains are light blue and lime. (B) Superposition of KR01 HA in Fab0587 complex and head domain of KR01 HA structures, and (C) superposition of stem region between Fab0587 bound KR01 HA and Free KR01 HA. Gray color represents free $\mathrm{HA}$, and orange color represents $\mathrm{HA}$ in complex. doi:10.1371/journal.pone.0089803.g002

chain CDRs (LCDRs) 1 and 3 (Fig. 3B). A total buried surface area at the interface in the Fab0587 complex is 1,029 $\AA^{2}$, of which $48 \%$ arise from binding to the $\mathrm{H}$-chain and $52 \%$ from the L-chain. In the Fab0757 complex, it is $993.2 \AA^{2}$, with $50 \%$ from the $\mathrm{H}$ - and $\mathrm{L}$-chains each. The buried surface areas are in the increasing order of LCDR1, HCDR2, LCDR3, and HCDR3, ranging from 230 to $300 \AA^{2}$. LCDR3 and HCDR3 have the largest number of interactions in the HA-Fab0587 complex, whereas LGDR3 and HCDR2 have the largest in the HA-Fab0587 complex (Table S1 in File $\mathrm{S} 1)$. It is notable that aromatic amino acid residues are found to contribute to the interactions at the interface between HA and Fab: Tyr31 of LCDR1, Tyr58 of HCDR2, Tyr99 of LCDR3, and Tyr103 of HCDR3 interact with residues Thr72, Lys163,
Glu115, and Lys171 of HA, respectively, in both complex structures (Fig. 3C).

Human $\kappa$ chain LCDR3 is typically nine amino acids long, whereas HCDR 3 lengths range from 6 to 28 amino acids in adults [31]. HCDR 3 and LCDR 3 of 6 to 8 residues in length in Fab0587 and Fab0757 represent a short-length CDR, but they contribute substantially to the interactions with HA molecules (Fig. 3C, inset). Glul12 and Glul15 have close contacts with the main chain atoms of Tyr 103 and Thr104 in HCDR3 and of Glu96 and Val97 in LCDR3, respectively. The side chain of Glul15 forms a hydrogen bond with the hydroxyl group of Tyr99. Lys169 and Lys 171 also contribute significantly to the interaction in HCDR3. These interactions revealed common features at the interface in 
Table 2. Data and refinement statistics.

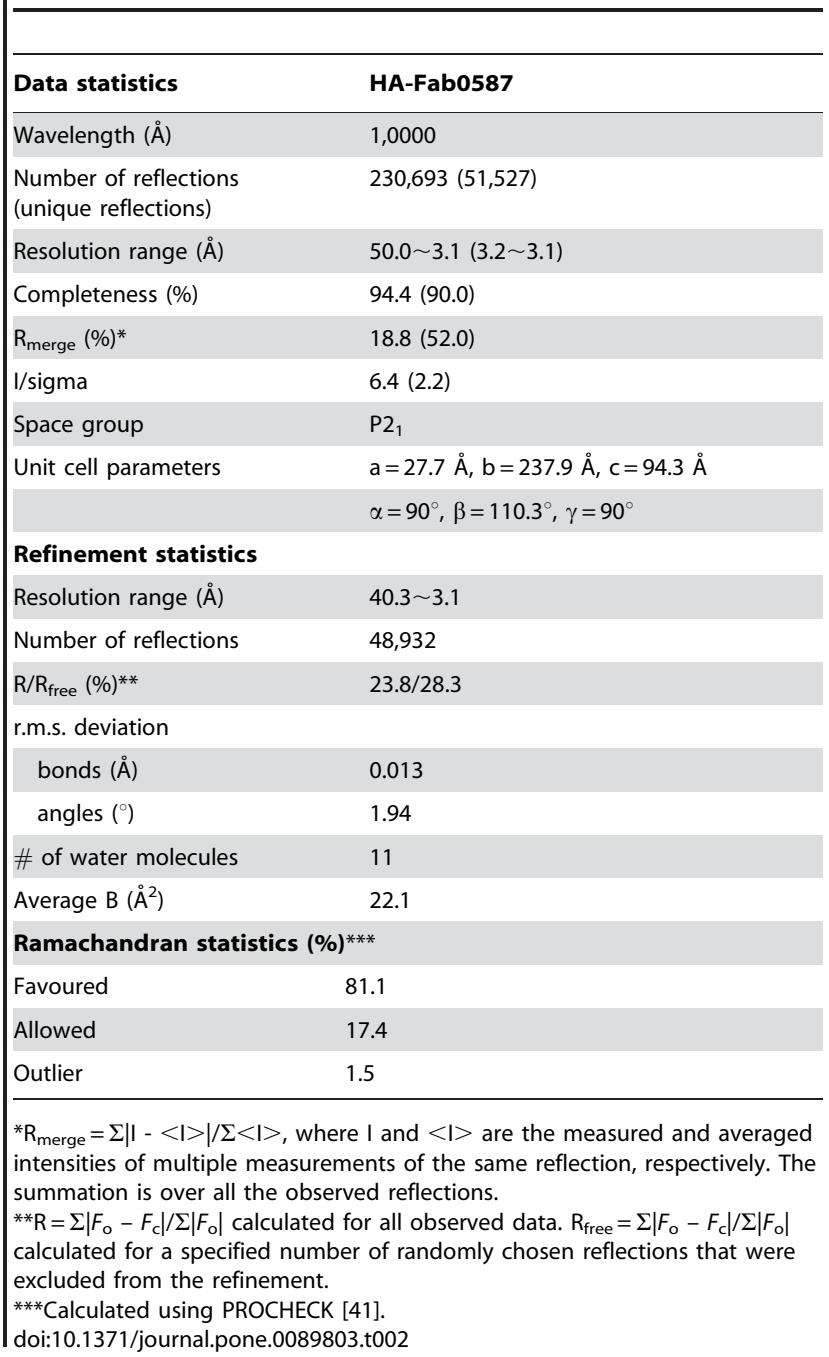

the complex structures. Both Glu112 and Glul15 are highly conserved in $\mathrm{H} 1$ subtype viruses, whereas they are not in other subtype HA sequences (Fig. $3 \mathrm{~A}$ and Figure S2 in File S1). In addition, Pro118, Ser121, Lys163, and Tyr165 in HA that interact with HCDR2, and Tyr253 that interacts with LCDR3 are highly conserved in $\mathrm{H} 1$ subtype strains. Notably, these residues are found to be different from those of the known antigenic sites, $\mathrm{Sa}, \mathrm{Sb}, \mathrm{Ca}$, and $\mathrm{Cb}$ (Fig. 3D). In this context, KR01 HA structures in complex with GC0587 or GC0757 reveal a distinct epitope in the head region which was not reported before.

\section{Interactions at the KR01 HA-Fab Complexes}

Both GC0587 and GC0757 neutralize a broad range of H1 subtype viruses including $2009 \mathrm{pdm} \mathrm{H} 1 \mathrm{Nl}$ and seasonal H1N1 strains, and GC0757 further reacts with 1918 pdm H1N1 (Table 1 and Fig. 1). Despite similar amino acid sequences and common structural features of the two Fab fragments, a detailed structural analysis of the HA-Fab0587 complex revealed several epitope regions distinct from those of the HA-Fab0757 complex. First, the main chain of HA from Thr72 to Ser74 moves closer to the Fab fragment in the Fab0587 complex and interacts mostly with LCDR1 from Asn30 to Ile33 (Figure S3 in File S1). There are relatively less hydrophilic interactions between HA and LCDR 1 in the Fab0587 complex than other CDRs. Second, the interactions
A
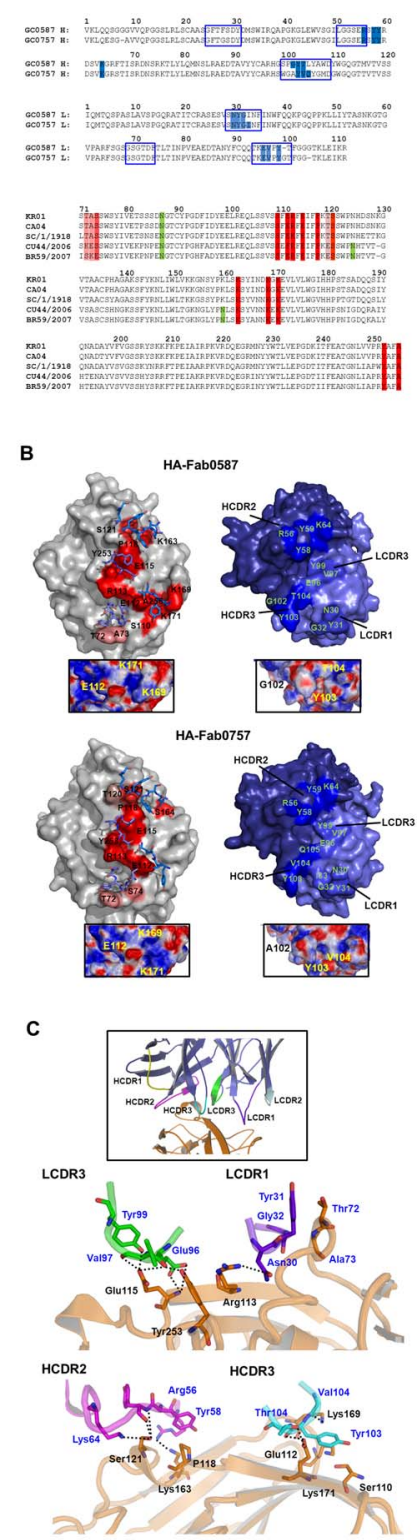

D

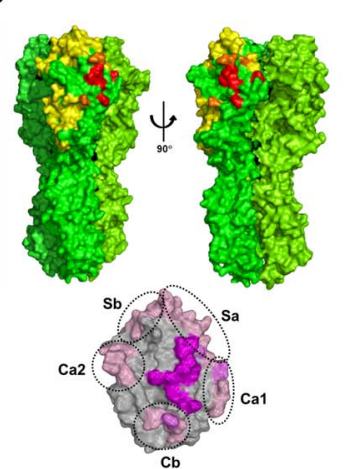

Figure 3. Sequence alignments and antigenic sites. (A) Sequence alignments of variable region of GC0587 and GC0757 (upper panel) and H1 HAs (lower panel). Residues in CDRs are in blue open boxes and residues that interact with $\mathrm{HA}$ are highlighted in blue filled boxes. Epitopes in $\mathrm{H} 1 \mathrm{HAs}$ are highlighted in pink, and more conserved residues are highlighted in red. Potential glycosylation sites are 
highlighted in green. (B) Surface representations of KR01 HAs (gray) and Fab fragments (dark blue and light blue for $\mathrm{H}$-chain and L-chain, respectively). Antigenic sites are colored in red for highly conserved residues and pink for moderately conserved residues. Amino acid residues involved in the interactions between KR01 HA and Fab0587 are colored in blue and slate. Insets are surface charge representations with contours from -10 (red) to +10 (blue) $k T$ through 0 (white). (C) Detailed interactions of $\mathrm{HA}$ with Fab0587. HA and Fab are colored in orange and blue, respectively. Residues that contribute to the interactions are represented as stick models. LCDR1 and LCDR3 are colored in purple and green, respectively (upper panel), and HCDR2 and HCDR3 are colored in magenta and cyan, respectively (lower panel). (D) Residues found at other structurally characterized antibody complexes are colored in yellow, those at both Fab0587 and other antibody complexes are in orange, and those against GC0587 are in red. Classical antigenic sites are colored in light pink $(\mathrm{Ca}, \mathrm{Cb}, \mathrm{Sa}$, and $\mathrm{Sb})$ and those at both Fab0587 antigenic sites are in pink.

doi:10.1371/journal.pone.0089803.g003

between HA and LCDR 3 are mainly mediated by Glul15 and Tyr253 of HA and Glu96 and Tyr99 of Fab (Fig. 4A). In the Fab0587 complex, the carboxylic group of Glu115 of HA interacts with the main chain atoms of Glu96 and Val97 and the hydroxyl group of Tyr99. In contrast, the main chain atoms of Arg113 and side chain atoms of Tyr253 interact with Glu96 in the Fab0757 complex. The side chain of Glu115 forms hydrogen bonds with the main chain nitrogen of Val97. In fact, the number of interactions by LCDR3 in the 757 complex is greater than that in the 587 complex (Table S1 in File S1).

Third, Arg56 of HCDR2 interacts with the side chain of Lys 163 of HA in the Fab0587 complex, while it interacts with the main chain of Ser164 of HA in the Fab0757 complex (Figure S4 in File S1). Tyr58 has hydrophobic contacts with Prol18 and the backbone atom of Tyr59 has hydrophilic interactions with Ser121 of HA in both complex structures. Fourth, the main differences between Fab0587 and Fab0757 reside in HCDR3, where only three residues (Tyr103, Tyr106, and Asp 109) are in common from residues 99 to 110 . The side chain of Glul12 of HA interacts with Thr104 in Fab0587 (Fig. 4B). In contrast, the side chain of Glul12 of HA interacts with the main chain oxygens of Tyr103 and Val104 in Fab0757. The interaction between Glul15 of HA and Gln105 of Fab is absent in the Fab0587 complex, whereas it is present in the Fab0757 complex due to Leu to Gln mutation. Notably, the side chain of Tyr103 in the Fab0587 complex is in a favourable arrangement for a cation- $\pi$ interaction with that of Lys171 of HA, and Lys169 has additional interactions with the main chain oxygen of Gly102 (Fig. 4C). However, the side chain of Tyr 103 protrudes outside from the surface in the Fab0757 complex, where the cation- $\pi$ interaction is absent. Both Lys169 and Lys 171 are highly conserved among $\mathrm{H} 1$ subtype viruses and adopt distinct conformations in each complex, interacting to different HCDR3 loop conformations.

\section{Epitope Specificity Based on HA Structures}

Both 2009 pdm KR01 HA and 1918 pdm HA exhibit 87\% pairwise sequence identity, showing $20 \%$ amino acid difference in the antigenic sites. However, antigenic and glycosylation patterns of 2009 pdm HA are rather similar to those of 1918 pdm HA [14]. In the Fab0587 complex, the presence of Prol01 in HCDR3 may play an important role in stabilizing the protrusion of Tyr 103 (Fig. 4C). Lys171 of HA is consequently able to make a cation- $\pi$ interaction with Tyr103. In contrast, the presence of Gly101 in HCDR 3 in the Fab0757 complex creates a space for the side chain of Lys 169 of HA to occupy, and the cation- $\pi$ interaction is absent. When the structures of KR01 HA and $1918 \mathrm{pdm}$ HA are superimposed, the side chains of Lys172(Lys 169) and
A

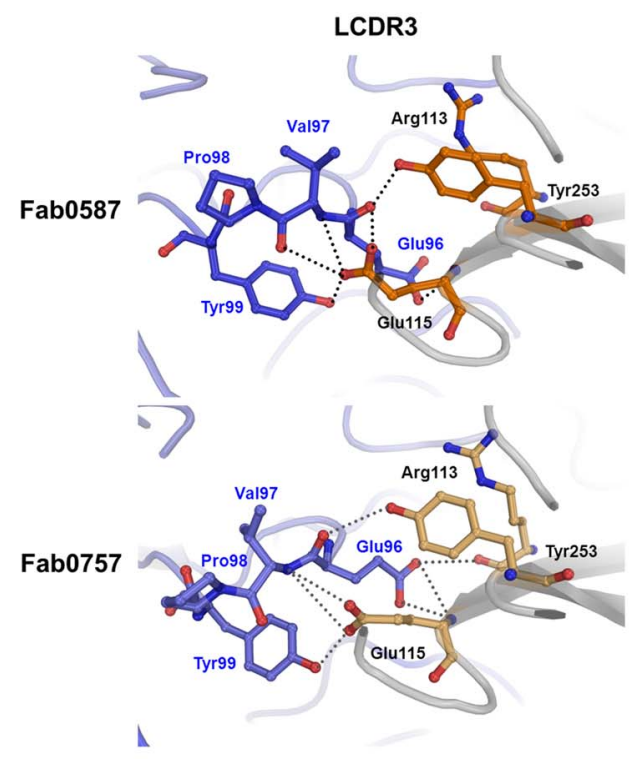

B

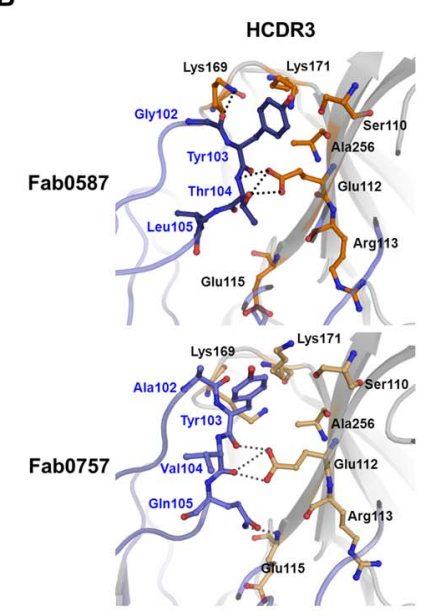

c

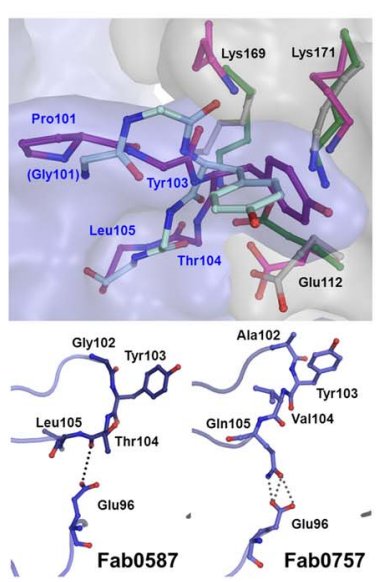

Figure 4. Comparison of Fab binding sites. Residues involved in interactions between HA and Fab0587 are represented as a ball-and- 
stick model and hydrophilic interactions as dotted line. Residues of HA in complex with Fab0587 are colored in orange and H-chains of Fab0587 and Fab0757 are in dark and light blue, respectively. (A) Comparison of LCDR3, (B) HCDR3. (C) Superposition of KR01 HA bound to Fab0587 (magenta), KR01 HA bound to Fab0757 (dark gray), free KR01 HA (yellow), 1918 pdm HA (green), Fab0587 (purple), and Fab0757 (light teal). Surface representations are based on the KR01HA-Fab0587 complex structure.

doi:10.1371/journal.pone.0089803.g004

Lys 174(Lys 171) of 1918 pdm HA are close to interact with Glu112. In the Fab0587 complex, however, they would not be compatible with the protruding conformation of HCDR3, whereas they can make their conformations complementary to HCDR 3 in the Fab0757 complex. Our results thus suggest that the conformation of the HCDR3 backbone in the Fab0587 complex is too close to interact with $1918 \mathrm{pdm} \mathrm{HA}$, causing steric hindrance. Tyr103 and Thr104 form hydrogen bonds with Glu96 of LCDR3. In contrast, Gln105 in Fab757 interacts with Glu96 of LCDR3 (Fig. 4C, lower panel). These interactions contribute to distinguished conformations between Fab0587 and Fab0757 complexes. Overall, our results strongly suggest that residues Ser110-Glu115, Lys169-Lys171, and Tyr253 of HA provide a H1-specific epitope and both HCDR3 and LCDR3 make major contributions to antibody-antigen interactions to achieve the epitope specificity.

\section{Discussion}

\section{Implication of KR01 HA-Fab Complexes}

The most effective way to protect against influenza virus infection is through vaccination. However, a good match between circulating strains and the isolates included in the vaccine is often difficult to attain due to rapid antigenic drift to evade existing antibody responses [32]. The introduction of $2009 \mathrm{pdm}$ influenza virus into the human population re-emphasized the need for a systematic approach to assessing immunogenic epitopes. A typical binding of antibodies to variable positions at the epitope due to antigenic drift renders them largely strain specific and susceptible to antigenic escape.

GC0587 and GC0757 share common epitopes that are conserved in $\mathrm{H} 1 \mathrm{Nl}$ strains, except for $1918 \mathrm{pdm}$ virus. The structural features of the complexes provided a critical understanding of how antibodies with subtype specificity can distinguish antigenic determinants. It was previously reported that CH65 which binds to receptor binding pocket of HA cross-neutralizes an unusually large subset of $\mathrm{H} 1 \mathrm{~N} 1$ viruses using five of the six CDR loops [23]. Of 27 contact residues of the HA-Fab0587 complex structure, 7 core residues are $>99 \%$ conserved across human $\mathrm{Hl}$ HA viruses: Glu112, Glu115, Pro118, Ser121, Lys163, Lys169, and Lys171. In particular, the conserved amino acid residues Glu112 and Glul15 have close contacts with HCDR3 and LCDR3, respectively. They thus comprise the epitopes recognized by both GC0587 and GC0757 and are different from the known antigenic sites, $\mathrm{Sa}, \mathrm{Sb}, \mathrm{Ca}$, and $\mathrm{Cb}$, exhibiting a distinct epitope in the head region for $\mathrm{Hl}$ subtype specificity. Notably, the new epitope is highly conserved among $\mathrm{Hl}$ influenza variants and has no glycosylation sites. It also reveals common features of interface contacts including HCDR3 and LCDR3, which explains that GC0587 and GC0757 have a broad cross-reactivity among H1 subtypes. H1 subtype-specific epitopes were recently identified by peptide scanning using libraries of overlapping peptides [33]. A new epitope (residues 58-72) was close to the link between the stem and globular head regions, which is highly conserved. Another epitope-mimic peptide (residues 158-182) that was highly immunogenic is close to what we observed in this study, where residues from Lys 169 to Lys 170 are included in the epitope mimic peptide. Taken together, unlike known $\mathrm{H} 1$ antigenic sites, the $\beta$ strands constituted by Ser110-Glu115 and Lys169-Lys 170 formed $\mathrm{H} 1$ epitopes with distinct conformations from those of $\mathrm{H} 3 \mathrm{HA}$. The backbone conformation of $\mathrm{H} 1 \mathrm{HA}$ was significantly different from that of $\mathrm{H} 2, \mathrm{H} 5$ and $\mathrm{H} 3 \mathrm{HAs}$ in roughly increasing order of deviation (Figure S4 in File S1). The 16 subtypes of HA segregate into two groups [7], where $\mathrm{H} 1$ and $\mathrm{H} 3$ belong to group 1 and 2, respectively. This epitope region was the most different in various subtype HAs and makes the largest difference in conformation between $\mathrm{H} 1$ and $\mathrm{H} 3 \mathrm{HAs}$.

Moreover, the main differences between Fab0587 and Fab0757 complex structures reside mainly in HCDR3 and LCDR3, although some conformational differences are found in HCDR2 and LCDR 1. It was shown that the antigenic pattern of $2009 \mathrm{pdm}$ HA is similar to those of 1918 pdm HA [14]. The interaction of Lys172(Lys169) and Lys174(Lys171) with Glul12 of 1918 pdm HA is not compatible with the protruding conformation of HCDR3 of Fab0587, whereas it can be compatible with that of Fab0757. Thus, Glu112, Lys169, and Lys171 are important epitope residues to differentiate Fab0587 and Fab0757, and HCDR 3 and LCDR3 make major contributions to antibodyantigen interactions to achieve epitope specificity. The unpredictability of the occurrence of influenza epidemics is originated from the wide range of antigenically different viruses, and the structural features of the complexes provide an understanding of how antibodies with subtype specificity can distinguish antigenic determinants. The results of our complex structures of a neutralizing monoclonal antibody with $\mathrm{Hl}$ subtype specificity can be used for potential use against influenza A viruses.

\section{Materials and Methods}

\section{Preparation of Cells}

MDCK cells (American Tissue Culture Type CCL-31) and Vero cells (Korean Cell Line Bank 10081) were grown in Dulbecco's modified Eagle's medium (DMEM, Invitrogen, USA) supplemented with 10\% fetal bovine serum (FBS, Sigma, USA), $1 \%$ glutamine, and $1 \%$ penicillin-streptomycin (PS, Invitrogen, USA) under $5 \% \mathrm{CO}_{2}$ humidified atmosphere at $37^{\circ} \mathrm{C}$. The cells were passed for no more than 20 passages.

\section{Virus Collection and Assays}

H1N1 A/Thailand/CU44/2006 (CU44), A/Brisbane/59/2007 (BR59) and 2009 pdm KR01, and H3N2 A/Gyeongnam/684/ 2006 (Gy684) were propagated in MDCK or Vero cells with DMEM supplemented with $0.2 \%$ BSA, $25 \mathrm{mM}$ HEPES, $1 \%$ glutamine, $1 \%$ penicillin-streptomycin, and $2 \mu \mathrm{g} / \mathrm{ml}$ of $\mathrm{L}$ (tosylamido-2-phenyl) ethyl chloromethyl ketone (TPCK)-treated trypsin (from bovine pancreas, PIERCE, USA) under $5 \% \mathrm{CO}_{2}$ atmosphere at $37^{\circ} \mathrm{C}$ for $2-3$ days. Virus titer was determined by using plaque assay $(\mathrm{PFU} / \mathrm{ml}), \mathrm{TCID}_{50}$, and hemagglutination assay and virus stocks were stored in deep freezer $\left(-77^{\circ} \mathrm{C}\right)$ until experiments.

Immunization and Production of Monoclonal Antibodies

Four-week old female Balb/c mice were immunized by intraperitoneal injection of $100 \mu \mathrm{g}$ of $2009 \mathrm{pdm} \mathrm{H1Nl} \mathrm{A}$ / California/04/2009 (CA04) vaccine dissolved in $100 \mu \mathrm{l}$ PBS plus complete Freund's adjuvant (Sigma-Aldrich, St Louis, MO, USA). Boost immunizations were given at 3 weeks post-vaccination. Animals were maintained and treated according to the Green Cross guidelines. All experiments in mice were approved by the 
Institutional Animal Care and Use Committee (IACUG) of the Green Cross.

Mouse spleen cells collected at 4 days after the 5 th immunization boost with vaccine were fused to the sp2/0 myeloma cells. Fused cells were distributed over 96 well tissue culture plates at 2,000 cells per well in complete DMEM medium containing $100 \mu \mathrm{M}$ hypoxanthine, $0.4 \mu \mathrm{M}$ aminopterin, and $16 \mu \mathrm{M}$ thymidine (HAT). With weekly replacement of medium, antibodyproducing cells were screened by ELISA and subcloned by limiting dilution. Ferret anti-BR59 whole serum was used as 1/50 dilution and monoclonal antibody was used at a final concentration of $80 \mu \mathrm{g} / \mathrm{ml}$. Positive clones were checked for isotype by using Iso-Gold ${ }^{\mathrm{TM}}$ rapid mouse-monoclonal isotyping test kit (Bioassay Works, Ijamsville, MD, USA) as described in the manufacturer's protocol.

Microtiter plates were coated with $100 \mu \mathrm{l}$ of vaccine or its equivalent $\mathrm{HA}(5 \mu \mathrm{g} / \mathrm{ml})$ diluted in PBS per well at $4{ }^{\circ} \mathrm{C}$ overnight, and blocked with PBS containing 1\% BSA and $0.05 \%$ Tween 20 at room temperature for $1 \mathrm{hr}$. One hundred $\mu \mathrm{l}$ of hybridoma supernatant or purified antibodies per well was transferred into the ELISA plates. Binding reaction was carried out at room temperature for $2 \mathrm{hrs}$. Plates were subsequently washed four times with PBS containing $0.05 \%$ Tween 20 , and $100 \mu \mathrm{l}$ of HRPconjugated goat anti-human Fab (Sigma-Aldrich, St Louis, MO, USA) diluted 1:10,000 in PBS was added, and reactions were carried out at room temperature for $1 \mathrm{hr}$. Plates were washed four times, and $100 \mu \mathrm{l}$ of tetramethyl benzidine substrate (KPL, Gaithersburg, MD, USA) per well was added. The absorbance was determined at $490 \mathrm{~nm}$.

\section{Cloning and Baculovirus Production}

HA gene were cloned downstream of the gp67 secretion signal sequence of the transfer vector pAcGP67A (BD Biosciences, MA, USA). Based on H3 numbering, the corresponding residues were 11-329 (1-327) (HAl) and 330-506 (1-176) (HA2), with a thrombin cleavage site, foldon region, and 6xHis-tag downstream of the HA gene sequence [34]. The recombinant HA proteins contained additional plasmid-encoded residues (ADPG for seasonal $\mathrm{Hl}$ and ADPGYLLEF for $2009 \mathrm{pdm} \mathrm{H1}$ ) at their $\mathrm{N}$ terminus and RSLVPR at the C-terminus. All sequences were confirmed by automated sequencing (Macrogen, Seoul, Korea). Plasmids encoding each HA gene were amplified in E. coli strain DH5 $\alpha$ and then used to co-transfect Sf9 cells along with linearized baculovirus chromosomal DNA (BaculoGold; BD Biosciences, MA, USA) by the calcium transfection method. The virus was harvested after 5 days, and transfection efficiency was confirmed by PCR after extraction of DNA from $400 \mu$ of virus (cell supernatant). This original virus stock was amplified through repeated rounds of infection.

\section{Protein Expression and Purification}

Baculovirus containing HA gene was used to infect suspension cultures of $\mathrm{Hi} 5$ cells. After $3 \sim 4$ days at $28^{\circ} \mathrm{C}$, the culture medium was harvested and applied to a Ni-NTA column equilibrated with working buffer (20 mM Tris-HCl, pH 8.0, $200 \mathrm{mM} \mathrm{NaCl}$ ). The column was washed with buffer containing $50 \mathrm{mM}$ imidazole and precursor HA protein was eluted in an imidazole gradient, which was dialysed against $10 \mathrm{mM}$ Tris- $\mathrm{HCl}(\mathrm{pH} 8.0)$ and $50 \mathrm{mM} \mathrm{NaCl}$, and hydrolysed by thrombin for $12 \mathrm{hrs}$ at $4^{\circ} \mathrm{C}$ for removal of the foldon region and $6 x$ His-tag. The reaction was halted by the addition of $1 \mathrm{mM}$ phenylmethylsulphonyl fluoride, and the active form of HA was purified by Mono $\mathrm{Q}$ ion-exchange chromatography and Superdex 200HR size exclusion chromatography.
The neutralizing antibodies with activity against $\mathrm{H} 1$ subtype influenza viruses, GC0346, GC0587, GC0757, and GC1517, were purified using protein A columns (GE Healthcare, Piscataway, NJ, USA). Each was then treated with papain at a ratio of 1:50 (w/w) for $2 \mathrm{hrs}$ in PBS buffer $\left(10 \mathrm{mM} \mathrm{Na}{ }_{2} \mathrm{HPO}_{4}, 1.8 \mathrm{mM} \mathrm{KH} \mathrm{PO}_{4}\right.$, $137 \mathrm{mM} \mathrm{NaCl}, 2.7 \mathrm{mM} \mathrm{KCl}$, and $\mathrm{pH}$ 7.4) with $5 \mathrm{mM}$ EDTA and $5 \mathrm{mM}$ L-cysteine, and the reaction was stopped with $30 \mathrm{mM}$ crystalline iodoacetamide. The solution containing Fab fragments was dialyzed against the PBS buffer and applied to protein A column. The unbound fraction was purified by Superdex 200HR gel filtration chromatography. It was then mixed with $\mathrm{HA}$ at a 1:1.5 molar ratio for $12 \mathrm{hr}$ incubation, and the complex was further purified by Superdex $200 \mathrm{HR}$ gel filtration chromatography.

\section{Crystallization and Data Collection}

The complex of HA and Fab0587 was screened by hanging drop vapour diffusion method. $1 \mu \mathrm{l}$ of the $10 \mathrm{mg} / \mathrm{ml}$ complex in $20 \mathrm{mM}$ Tri-HCl (pH 8.0) and $50 \mathrm{mM} \mathrm{NaCl}$ was mixed with $1 \mu \mathrm{l}$ of screening solution of $20 \%$ PEG3350 and $200 \mathrm{mM}$ potassium iodide and incubated at $4^{\circ} \mathrm{C}$. After one week, small rhombic crystals were obtained. Diffraction data were collected with the crystals flash-cooled at $100 \mathrm{~K}$ in a stream of liquid $\mathrm{N}_{2}$ in the mother liquor containing $22 \%$ glycerol using synchrotron radiation sources at beamlines 5C at Pohang Light Source (Pohang, Korea). The crystals of HA-Fab0587 diffracted to $2.8 \AA$ resolution, which belong to space group $\mathrm{P} 21$ with unit cell dimensions $\mathrm{a}=72.8 \AA, \mathrm{b}=237.8 \AA, \mathrm{c}=94.2 \AA, \alpha=90^{\circ}, \beta=110.3^{\circ}, \gamma=90^{\circ}$ (Table 1). All data were processed and scaled using the HKL2000 program [35].

\section{Structure Solution and Refinement}

The crystal structure of KR01 HA-Fab0587 was solved by molecular replacement using the KR01 HA-Fab0757 structure (PDB ID 4F15) as a template using CCP4 [36] or PHENIX [37]. The solvent content of HA-Fab0587 crystals were $65.2 \%$ using Matthews coefficient of 2.87, indicating that four complexes of HA and Fab fragments exist in the asymmetric unit. After the substitution of the Fab sequences with those of Fab0587 and manual adjustment using Coot [38], the initial solution was optimized by rigid body refinement, which produced interpretable electron density maps for the overall structure. Manual adjustment of the backbone and side chains was conducted and crystallographic refinement was carried out using the program PHENIX [37]. Difference Fourier maps, 2|Fo $|-| F_{c} \mid$ and $\left|F_{0}\right|-\left|F_{c}\right|$, have been used to model the active site or loop regions. After a few rounds of model rebuilding, water molecules were added using the $\mathrm{F}_{\mathrm{o}}-\mathrm{F}_{\mathrm{c}}$ map peaks above $3.0 \sigma$, if the $\mathrm{B}$ factors were below $50 \AA^{2}$ after refinement. The $\mathrm{R}_{\text {free }}$ value was used as an indicator to validate the water picking and refinement procedure and to guard against possible overfitting of the data [39]. $\mathrm{R}$ factor and $\mathrm{R}$ free were 0.23 and 0.27 , respectively, after several round of refinement using PHENIX program. Data quality and refinement statistics for both Fab0587-HA and Fab0757-HA complexes are presented in Table 2. Stereochemical analysis of all refined structures using PROCHECK [40]. showed that there were $1.6 \%$ of outliers in the Ramachandran plot with $81.2 \%$ of favoured region among the 2,577 residues for HA-Fab0587. These outliers were located in the disordered loop regions.

\section{Accession Number}

Data deposition-The atomic coordinates and structure factors have been deposited in the Protein Data Bank (www.rcsb.org) with PDB ID code 4LVH for H1N1 HA-Fab0587 structure. 


\section{Supporting Information}

File S1 Supporting Information. Table S1, Interactions between KR01 HA and Fab fragments. Figure S1, Crystal packing in the KR01 HA-Fab0587 complex. The head domain (HAl) of $\mathrm{HA}$ is shown in red color and Fab in blue in one complex, and others are in green. The stem regions (HA2) of HA could not be modeled due to weak electron density, but there are empty space for them. A partial model for HA2 is shown in the lower panel. Figure S2, Sequence alignments of HAs. Epitopes in H1 HAs are highlighted in red, and more conserved residues are highlighted in more red. Residues in other HA those are same with that of epitope in $\mathrm{Hl}$ HAs are indicated by red boxes. Potential glycosylation sites are highlighted in green. Figure S3, LCDR1 at Fab binding site. Residues involved in interactions between HA and Fab0587 are represented as a ball-and-stick model and hydrophilic interactions as dotted line. Residues of HA in complex with Fab0587 are colored in orange and H-chains of Fab0587 and Fab0757 are in dark and light blue, respectively. Figure S4,

\section{References}

1. KwongJC, Stukel TA, Lim J, McGeer AJ, Upshur RE, et al. (2008) The effect of universal influenza immunization on mortality and health care use. PLoS Med 5: e211.

2. Dushoff J, Plotkin JB, Viboud C, Earn DJ, Simonsen L (2006) Mortality due to influenza in the United States-an annualized regression approach using multiple-cause mortality data. Am J Epidemiol 163: 181-187.

3. Johnson NP, Mueller J (2002) Updating the accounts: global mortality of the 1918-1920 "Spanish" influenza pandemic. Bull Hist Med 76: 105-115.

4. WHO.

5. Cox NJ, Subbarao K (2000) Global epidemiology of influenza: past and present. Annu Rev Med 51: 407-421.

6. Osterhaus AD, Rimmelzwaan GF, Martina BE, Bestebroer TM, Fouchier RA (2000) Influenza B virus in seals. Science 288: 1051-1053.

7. Gamblin SJ, Skehel JJ (2010) Influenza hemagglutinin and neuraminidase membrane glycoproteins. J Biol Chem 285: 28403-28409.

8. Pielak RM, Chou JJ (2011) Influenza M2 proton channels. Biochim Biophys Acta 1808: 522-529.

9. Rohm C, Zhou N, Suss J, Mackenzie J, Webster RG (1996) Characterization of a novel influenza hemagglutinin, H15: criteria for determination of influenza A subtypes. Virology 217: 508-516.

10. Zhu X, Yu W, McBride R, Li Y, Chen LM, et al. (2013) Hemagglutinin homologue from $\mathrm{H} 17 \mathrm{~N} 10$ bat influenza virus exhibits divergent receptorbinding and $\mathrm{pH}$-dependent fusion activities. Proc Natl Acad Sci U S A 110: 1458-1463.

11. Taubenberger JK, Reid AH, Lourens RM, Wang R, Jin G, et al. (2005) Characterization of the 1918 influenza virus polymerase genes. Nature 437: 889-893.

12. Neumann G, Kawaoka $Y$ (2006) Host range restriction and pathogenicity in the context of influenza pandemic. Emerg Infect Dis 12: 881-886.

13. Zimmer SM, Burke DS (2009) Historical perspective-Emergence of influenza A (H1N1) viruses. N Engl J Med 361: 279-285.

14. Xu R, Ekiert DC, Krause JC, Hai R, Crowe JE Jr, et al. (2010) Structural basis of preexisting immunity to the $2009 \mathrm{H} 1 \mathrm{~N} 1$ pandemic influenza virus. Science 328: 357-360.

15. Gething MJ, Doms RW, York D, White J (1986) Studies on the mechanism of membrane fusion: site-specific mutagenesis of the hemagglutinin of influenza virus. J Cell Biol 102: 11-23.

16. Copeland CS, Zimmer KP, Wagner KR, Healey GA, Mellman I, et al. (1988) Folding, trimerization, and transport are sequential events in the biogenesis of influenza virus hemagglutinin. Cell 53: 197-209.

17. Hebert DN, Foellmer B, Helenius A (1995) Glucose trimming and reglucosylation determine glycoprotein association with calnexin in the endoplasmic reticulum. Cell 81: 425-433.

18. Steinhauer DA (1999) Role of hemagglutinin cleavage for the pathogenicity of influenza virus. Virology 258: 1-20.

19. Klenk HD, Rott R, Orlich M, Blodorn J (1975) Activation of influenza A viruses by trypsin treatment. Virology 68: 426-439.

20. Okuno Y, Isegawa Y, Sasao F, Ueda S (1993) A common neutralizing epitope conserved between the hemagglutinins of influenza A virus $\mathrm{H} 1$ and $\mathrm{H} 2$ strains. J Virol 67: 2552-2558.
HCDR2 at Fab binding site. Residues involved in interactions between HA and Fab0587 are represented as a ball-and-stick model and hydrophilic interactions as dotted line. Residues of HA in complex with Fab0587 are colored in orange and H-chains of Fab0587 and Fab0757 are in dark and light blue, respectively. Figure S5, Comparison of novel $\mathrm{H} 1$ specific epitope regions among different subtypes. Superposition of the novel epitope conformations of $\mathrm{H} 1$ (blue), H2 (gray), H3 (dark gray), and H5 (green) HA. RMSD was calculated based on secondary structure matching of head domain of $\mathrm{Hl}$ with that of other subtypes, to show 1.19, 1.68 and $1.34 \AA$ for $\mathrm{H} 2, \mathrm{H} 3$ and $\mathrm{H} 5$, respectively. (DOCX)

\section{Author Contributions}

Conceived and designed the experiments: KJC KHK. Performed the experiments: KWH SHK SK JHS JL. Analyzed the data: KWH SHK SK JHS JL KJC KHK. Contributed reagents/materials/analysis tools: KJC XS KHK. Wrote the paper: KJC KHK.

21. Ekiert DC, Friesen RH, Bhabha G, Kwaks T, Jongeneelen M, et al. (2011) A highly conserved neutralizing epitope on group 2 influenza A viruses. Science 333: 843-850.

22. Corti D, Voss J, Gamblin SJ, Codoni G, Macagno A, et al. (2011) A neutralizing antibody selected from plasma cells that binds to group 1 and group 2 influenza A hemagglutinins. Science 333: 850-856.

23. Whittle JR, Zhang R, Khurana S, King LR, Manischewitz J, et al. (2011) Broadly neutralizing human antibody that recognizes the receptor-binding pocket of influenza virus hemagglutinin. Proc Natl Acad Sci U S A 108: 1421614221.

24. Sui J, Hwang WC, Perez S, Wei G, Aird D, et al. (2009) Structural and functional bases for broad-spectrum neutralization of avian and human influenza A viruses. Nat Struct Mol Biol 16: 265-273.

25. Mueller M, Renzullo S, Brooks R, Rugoli N, Hofmann MA (2010) Antigenic characterization of recombinant hemagglutinin proteins derived from different avian influenza virus subtypes. PLoS One 5: e9097.

26. Kaverin NV, Rudneva IA, Govorkova EA, Timofeeva TA, Shilov AA, et al. (2007) Epitope mapping of the hemagglutinin molecule of a highly pathogenic H5N1 influenza virus by using monoclonal antibodies. J Virol 81: 12911-12917.

27. Vareckova E, Cox N, Klimov A (2002) Evaluation of the subtype specificity of monoclonal antibodies raised against $\mathrm{H} 1$ and $\mathrm{H} 3$ subtypes of human influenza $\mathrm{A}$ virus hemagglutinins. J Clin Microbiol 40: 2220-2223.

28. Cho KJ, Lee JH, Hong KW, Kim SH, Park Y, et al. (2013) Insight into structural diversity of influenza virus hemagglutinin. J Gen Virol. 94: 1712 1722.

29. Skehel JJ, Wiley DC (2000) Receptor binding and membrane fusion in virus entry: the influenza hemagglutinin. Annu Rev Biochem 69: 531-569.

30. Harrison SC (2008) Viral membrane fusion. Nat Struct Mol Biol 15: 690-698.

31. Volpe JM, Kepler TB (2008) Large-scale analysis of human heavy chain V(D)J recombination patterns. Immunome Res 4: 3.

32. Salzberg S (2008) The contents of the syringe. Nature 454: 160-161.

33. Zhao R, Gui S, Guo L, Wu C, Gonzalez R, et al. (2011) Identification of a highly conserved $\mathrm{H} 1$ subtype-specific epitope with diagnostic potential in the hemagglutinin protein of influenza A virus. PLoS One 6: e23374.

34. Bhardwaj A, Walker-Kopp N, Wilkens S, Cingolani G (2008) Foldon-guided self-assembly of ultra-stable protein fibers. Protein Sci 17: 1475-1485.

35. Otwinowski Z, Minor W (1997) Processing of X-ray diffraction data collected in oscillation mode. Macromolecular Crystallography, Pt A 276: 307-326.

36. (1994) The CCP4 suite: programs for protein crystallography. Acta Crystallogr D Biol Crystallogr 50: 760-763.

37. Adams PD, Afonine PV, Bunkoczi G, Chen VB, Davis IW, et al. (2010) PHENIX: a comprehensive Python-based system for macromolecular structure solution. Acta Crystallogr D Biol Crystallogr 66: 213-221.

38. Emsley P, Cowtan K (2004) Coot: model-building tools for molecular graphics. Acta Crystallogr D Biol Crystallogr 60: 2126-2132.

39. Brunger AT (1992) Free R value: a novel statistical quantity for assessing the accuracy of crystal structures. Nature 355: 472-475.

40. Laskowski RA, Moss DS, Thornton JM (1993) Main-chain bond lengths and bond angles in protein structures. J Mol Biol 231: 1049-1067.

41. Laskowski RA, MacArthus MW, Moss DS, Thornton JM (1993) PROCHECK: a program to check the stereochemical quality of protein structures. J Appl Cryst 26: 283-291. 